\title{
Observation of collective excitation of two individual atoms in the Rydberg blockade regime
}

\author{
Alpha Gaëtan1, Yevhen Miroshnychenko1, Tatjana Wilk1, Amodsen Chotia², Matthieu Viteau², \\ Daniel Comparat ${ }^{2}$, Pierre Pillet ${ }^{2}$, Antoine Browaeys ${ }^{1 \star}$ and Philippe Grangier ${ }^{1}$
}

When two quantum systems interact strongly with each other, their simultaneous excitation by the same driving pulse may be forbidden. The phenomenon is known as blockade of excitation. Recently, extensive studies have been devoted to the so-called Rydberg blockade between neutral atoms, which appears when the atoms are in highly excited electronic states, owing to the interaction induced by the accompanying large dipole moments. Rydberg blockade has been proposed as a basic tool in quantum-information processing with neutral atoms ${ }^{1-5}$, and can be used to deterministically generate entanglement of several atoms. Here, we demonstrate Rydberg blockade between two atoms individually trapped in optical tweezers at a distance of $4 \mu \mathrm{m}$. Moreover, we show experimentally that collective two-atom behaviour, associated with the excitation of an entangled state between the ground and Rydberg levels, enhances the allowed single-atom excitation. These observations should be a crucial step towards the deterministic manipulation of entanglement of two or more atoms, with possible implications for quantum-information science, as well as for quantum metrology, the study of strongly correlated systems in many-body physics, and fundamental studies in quantum physics.

A large experimental effort is nowadays devoted to the production of entanglement, that is quantum correlations, between individual quantum objects such as atoms, ions, superconducting circuits, spins or photons. There are several ways to engineer entanglement in a quantum system. Here, we focus on a method that relies on a blockade mechanism where the strong interaction between different parts of a system prevents their simultaneous excitation by the same driving pulse. Single excitation is still possible but is delocalized over the whole system, and results in the production of an entangled state. This approach to entanglement is deterministic and can be used to realize quantum gates ${ }^{1}$ or to entangle mesoscopic ensembles, provided that the blockade is effective over the whole sample ${ }^{2}$. Blockade effects have been observed in systems where interactions are strong such as systems of electrons using the Coulomb force ${ }^{6}$ or the Pauli effective interaction $^{7}$, as well as with photons and atoms coupled to an optical cavity ${ }^{8}$. Recently, atoms held in the ground state of the wells of an optical lattice have been shown to exhibit interaction blockade, due to $s$-wave collisions ${ }^{9}$. An alternative approach uses the comparatively strong interaction between two atoms excited to Rydberg states. This strong interaction gives rise to the so-called Rydberg blockade, which has been observed in clouds of cold atoms $s^{10-15}$ as well as in a Bose condensate ${ }^{16}$. A collective behaviour associated with the blockade has been reported in an ultracold atomic cloud ${ }^{17}$. Recently, an experiment demonstrated the blockade

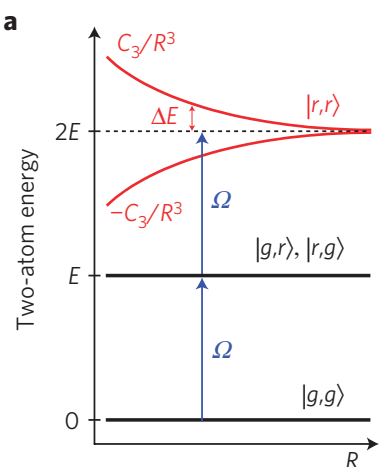

b



Figure 1 | Rydberg excitation of two atoms in the blockade regime. a, Principle of the Rydberg blockade between two atoms separated by a distance $R$. Two states $|g\rangle$ and $|r\rangle$ are coupled with Rabi frequency $\Omega$. When the two atoms are in state $|r, r\rangle$, they interact strongly, which leads to symmetrical energy shifts $\Delta E= \pm\left(C_{3} / R^{3}\right)$. When this shift becomes larger than $\hbar \Omega$, the laser is out of resonance with the transition coupling the singly and doubly excited states, and only one atom at a time can be transferred to the Rydberg state. $\mathbf{b}$, When the atoms are in the blockade regime, the state $\left|\Psi_{+}\right\rangle$, described in the text, is only coupled to the ground state $|g, g\rangle$ with a strength $\sqrt{2} \Omega$, whereas the state $\left|\Psi_{-}\right\rangle$is not coupled by the laser to the states $|g, g\rangle$ and $|r, r\rangle$. The atoms are therefore described by an effective two-level system.

between two atoms $10 \mu \mathrm{m}$ apart, by showing that when one atom is excited to a Rydberg state, the excitation of the second one is greatly suppressed ${ }^{18}$. However, the enhancement of the excitation rate of one atom when two atoms are present, explained by the excitation of an entangled state in the blockade regime, has not been observed until now.

Here, we study two individual atoms, held at a few micrometres distance by two optical tweezers. The ground state $|g\rangle$ and a Rydberg state $|r\rangle$ of an atom are separated by an energy $E$ (see Fig. 1a) and can be coupled by a laser. For non-interacting atoms, $a$ and $b$, the two-atom spectrum exhibits two transitions at the same frequency $E / \hbar$, connecting states $|g, g\rangle$ to $|r, g\rangle$ or $|g, r\rangle$, and then to $|r, r\rangle$. This enables the simultaneous excitation of the two atoms to state $|r, r\rangle$. However, if the two atoms interact strongly when in state $|r, r\rangle$, this energy level is shifted by an amount $\Delta E$ and the laser excitation cannot bring the two atoms to state $|r, r\rangle$. A fundamental consequence of this blockade is that the atoms are excited in the entangled state $\left|\Psi_{+}\right\rangle=(1 / \sqrt{2})\left(\mathrm{e}^{i \mathbf{k} \cdot \mathbf{r}_{a}}|r, g\rangle+\mathrm{e}^{i \mathbf{k} \cdot \mathbf{r}_{b}}|g, r\rangle\right)$, where $\mathbf{r}_{a}$ and $\mathbf{r}_{b}$ are the positions of the two atoms and $\mathbf{k}$ is related to the wave vectors of the exciting lasers. More

${ }^{1}$ Laboratoire Charles Fabry, Institut d'Optique, CNRS, Univ Paris-Sud, Campus Polytechnique, RD 128, 91127 Palaiseau cedex, France, ${ }^{2}$ Laboratoire Aimé Cotton, CNRS, Univ Paris-Sud, Bâtiment 505, Campus d'Orsay, 91405 Orsay cedex, France. *e-mail: antoine.browaeys@institutoptique.fr. 


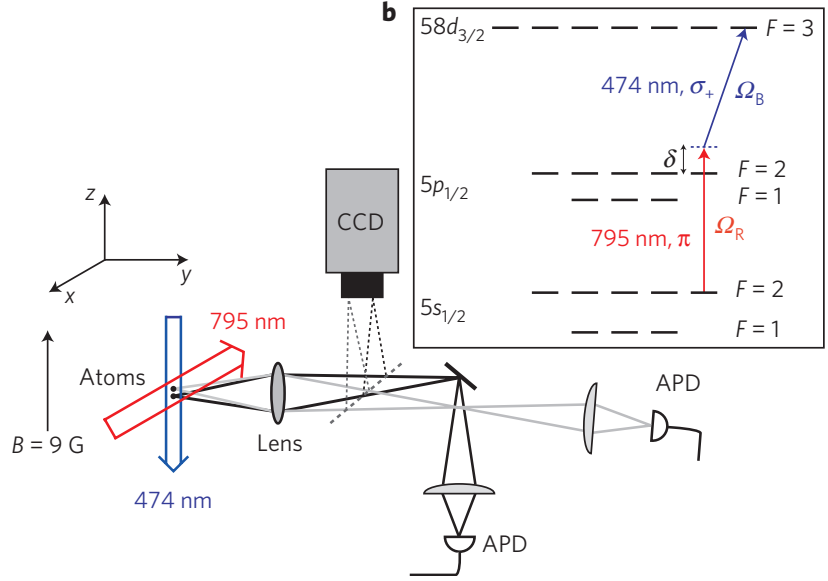

Figure 2 | Experimental details. a, Two rubidium 87 atoms are trapped in two tightly focused dipole traps (not shown). The quantization axis is set by a $9 \mathrm{G}$ magnetic field. The fluorescence of each trap is collected on two separated photon-counting avalanche photodiodes (APD). We can also image the two atoms on a CCD (charge-coupled device) camera, which enables us to measure the distance between the atoms. $\mathbf{b}$, The atoms are excited to the Rydberg state by a two-photon transition. An infrared laser at $795 \mathrm{~nm}$ propagating along the $x$ axis, with $\pi$-polarization, couples states $\left|5 s_{1 / 2}, F=2, M_{\mathrm{F}}=2\right\rangle$ and $\left|5 p_{1 / 2}, F=2, M_{\mathrm{F}}=2\right\rangle$. The laser is detuned to the blue side of this transition by $\delta=400 \mathrm{MHz}$, with a beam waist of $130 \mu \mathrm{m}$ and a power of $7 \mathrm{~mW}$. The second laser at $474 \mathrm{~nm}$ is $\sigma_{+}$-polarized, coupling the states $\left|5 p_{1 / 2}, F=2, M_{\mathrm{F}}=2\right\rangle$ and $\left|58 d_{3 / 2}, F=3, M_{\mathrm{F}}=3\right\rangle$, and propagates along the $z$ axis. It is focused on $25 \mu \mathrm{m}$ with $30 \mathrm{~mW}$ power. From a light-shift measurement, we get a Rabi frequency $\Omega_{\mathrm{R}} \approx 2 \pi \times 260 \mathrm{MHz}$. The two-photon Rabi frequency, measured experimentally (see Fig. 3), is given by $\Omega=\left(\Omega_{\mathrm{R}} \Omega_{\mathrm{B}} / 2 \delta\right)$, leading to $\Omega_{\mathrm{B}} \approx 2 \pi \times 21 \mathrm{MHz}$.

precisely, the laser excitation is described by the operator $(\hbar \Omega / 2)\left(\mathrm{e}^{\mathrm{ik} \cdot \boldsymbol{r}_{a}}|r, g\rangle\left\langle g, g\left|+\mathrm{e}^{\mathrm{ik} \cdot r_{b}}\right| g, r\right\rangle\langle g, g|+\right.$ complex conjugate $)$ (ref. 19). Here, $\Omega$ is the Rabi frequency characterizing the coupling between the laser and one atom. It is then convenient to use as a basis the two entangled states $\left|\Psi_{ \pm}\right\rangle=(1 / \sqrt{2})\left(\mathrm{e}^{i \mathbf{k} \cdot r_{a}}|r, g\rangle \pm \mathrm{e}^{\mathbf{k} \cdot \mathbf{r}_{b}}|g, r\rangle\right)$, so that $\left|\Psi_{-}\right\rangle$is not coupled to the ground state, whereas $\left|\Psi_{+}\right\rangle$is coupled with an effective Rabi frequency $\sqrt{2} \Omega$. In the blockade regime, where the state $|r, r\rangle$ is out of resonance, the two atoms are therefore described by an effective two-level system involving collective states $|g, g\rangle$ and $\left|\Psi_{+}\right\rangle$coupled with a strength of $\sqrt{2} \Omega$, as shown in Fig. 1b. Hence, the atoms are excited into an entangled state containing only one excited atom, with a probability oscillating $\sqrt{2}$ times faster than the probability to excite one atom when it is alone.

In our experiment, we excite two individual rubidium 87 atoms to the Rydberg state $|r\rangle=\left|58 d_{3 / 2}, F=3, M_{\mathrm{F}}=3\right\rangle$, with the internuclear axis aligned along the quantization axis. The state $58 d_{3 / 2}$ was chosen because of the existence of a quasi-degeneracy between the two-atom states $\left(58 d_{3 / 2}, 58 d_{3 / 2}\right)$ and $\left(60 p_{1 / 2}, 56 f_{5 / 2}\right)$, also called a Förster resonance ${ }^{20}$. As detailed in Supplementary Information, the dipole-dipole interaction lifts the degeneracy and leads to two potential curves $\pm\left(C_{3} / R^{3}\right)$, as represented in Fig. 1a. For our particular geometry, we calculated $C_{3} \approx h \times 3,200 \mathrm{MHz} \mu \mathrm{m}^{3}$. Accordingly, the interaction energy between two atoms at distance $R=4 \mu \mathrm{m}$ is $\Delta E \approx h \times 50 \mathrm{MHz}$.

The two single rubidium 87 atoms are confined in two independent optical dipole traps, as shown in Fig. 2a. The traps are formed in the focal plane of the same large numerical aperture lens ${ }^{21}$. Each trap has a waist of $0.9 \mu \mathrm{m}$ and a depth of $0.5 \mathrm{mK}$. The distance between the two traps can be varied between 3 and $20 \mu \mathrm{m}$ with a precision of $0.5 \mu \mathrm{m}$. The axis between the two traps is aligned with the magnetic field defining the quantization axis. The traps are
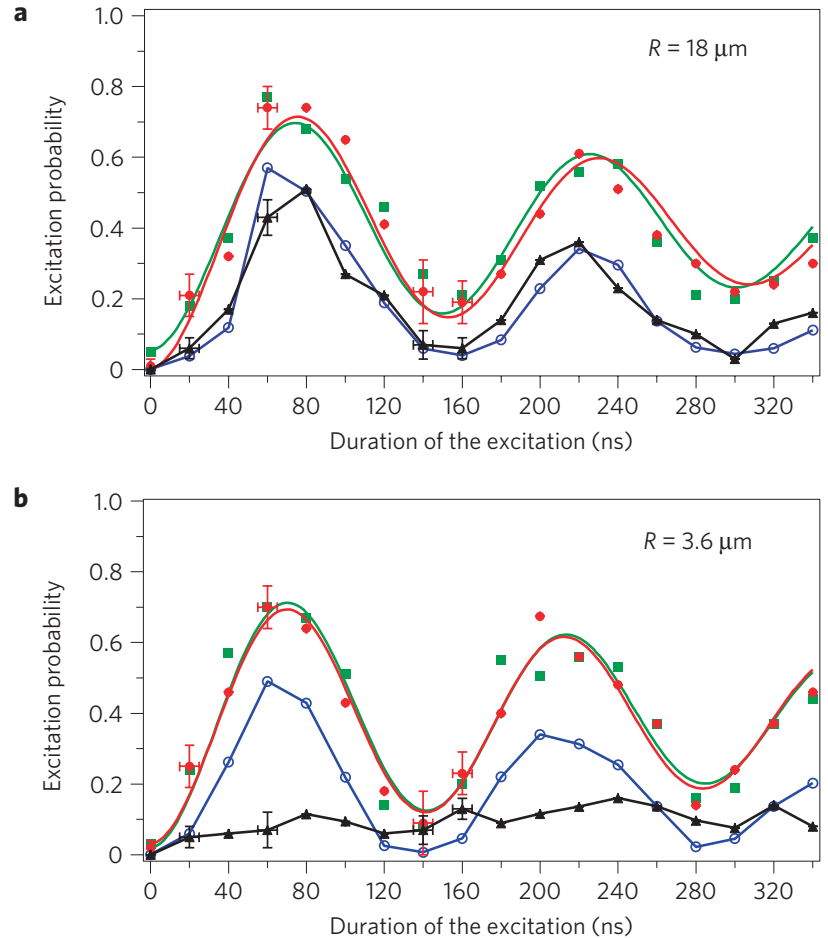

Figure $\mathbf{3}$ | Rydberg excitation of one and two atoms. In $\mathbf{a}$ and $\mathbf{b}$, the red circles and the green squares represent the probability to excite atom $a$ and atom $b$ respectively, when the other atom is absent. We fit the data by the function $A-B e^{-\frac{t}{\tau}} \cos \Omega t$, shown as solid red and green lines. The error bars on the data are the r.m.s. statistical error on the measured probability, as well as the error in the estimation of the pulse duration. The blue open circles are the product of the probabilities to excite atom $a$ and atom $b$ when the other one is absent. The triangles are the probability to excite the two atoms simultaneously when they are driven by the same pulse.

a, Atoms separated by $18 \mu \mathrm{m}$. The frequencies of the Rabi oscillations are 6.5 and $6.4 \mathrm{MHz}$ for atom $a$ and $b$ respectively. The agreement between the triangles and the blue circles indicates that the atoms do not interact.

b, Blockade of the Rydberg excitation when the two atoms are separated by $3.6 \mu \mathrm{m}$. Owing to the interaction between the atoms, this double excitation is greatly suppressed.

loaded from the cold atomic cloud of an optical molasses. We collect the fluorescence emitted by the atoms, induced by the cooling lasers, on two separated single-photon counters. A high fluorescence level indicates the presence of an atom in the respective trap and triggers the experimental sequence, described hereafter.

We prepare the two atoms in the hyperfine state $|g\rangle=\mid 5 s_{1 / 2}$, $\left.F=2, M_{\mathrm{F}}=2\right\rangle$ by a $600 \mu$ s optical pumping phase (efficiency $\sim 90 \%)$. We then excite the atoms to the Rydberg state $|r\rangle=\left|58 d_{3 / 2}, F=3, M_{\mathrm{F}}=3\right\rangle$ by a two-photon transition, as represented in Fig. $2 \mathrm{~b}$. The intermediate state $\left|5 p_{1 / 2}, F=2, M_{\mathrm{F}}=2\right\rangle$ is connected to the ground state by a laser detuned by $400 \mathrm{MHz}$ to the blue side of the $795 \mathrm{~nm}$ transition to avoid populating the intermediate state. The second laser connects the $5 p_{1 / 2}$ to the $58 d_{3 / 2}$ state and has a wavelength of $474 \mathrm{~nm}$. Both laser beams illuminate the two atoms. During the excitation ( $<500 \mathrm{~ns})$, the dipole trap is turned off to avoid an extra light-shift on the atoms. We finally detect the excitation to the Rydberg state through the loss of the atom after turning the dipole trap back on. In a Rydberg state, the atom is not trapped by the optical potential any longer. Owing to its residual velocity $\left(\sim 10 \mathrm{~cm} \mathrm{~s}^{-1}\right)$ it leaves the trapping region in less than $10 \mu$ s. This time is much shorter than the lifetime of Rydberg state $58 d_{3 / 2}$ induced by blackbody radiation ( $\left.160 \mu \mathrm{s}\right)$ and than the radiative decay time (200 $\mu$ s; ref. 22$)$. 
In a first experiment, we placed the two traps at a distance of $18 \pm 0.5 \mu \mathrm{m}$ and repeated the excitation sequence 100 times, starting each time with newly trapped atoms. We measured for each atom whether it was lost or recaptured at the end of each sequence and calculated the probability to excite it in the Rydberg state, which is equal to the probability to lose it. When only one of the two traps is filled, the excitation probability exhibits Rabi oscillations between the ground state and the Rydberg state, as shown in Fig. 3a. A fit to the data yields a two-photon Rabi frequency $\Omega \approx 2 \pi \times 7 \mathrm{MHz}$, which is in agreement with the measured waists and powers of the lasers. The decay of the fringe amplitude is explained by frequency fluctuations $(\sim 1 \mathrm{MHz})$ as well as shot-to-shot intensity fluctuations of the lasers $(\sim 5 \%)$, which results in a jitter in the twophoton resonance frequency. We attribute the maximum excitation probability of $\sim 80 \%$ to this decay and to the imperfect optical pumping of the atoms in the Zeeman state $\left|5 s_{1 / 2}, F=2, M_{\mathrm{F}}=2\right\rangle$.

We then repeated the sequence with two atoms trapped at the same time and measured the probability to excite the two atoms with the same laser pulse. The results are represented in Fig. 3a by the triangles. We compared this probability with the probability to excite simultaneously two non-interacting atoms, which should be equal to the product of the probabilities to excite each atom independently, measured previously. The blue circles in Fig. 3a represent this product, calculated from the data for each independent atom. The agreement between the two curves shows that the two atoms, when separated by $18 \mu \mathrm{m}$, behave independently and therefore have a negligible interaction. This result agrees with the theory because the blockade becomes effective at a distance between the atoms for which the interaction shift $\Delta E$ is equal to the linewidth of the excitation pulse, of the order of the Rabi frequency $\Omega$. For our particular choice of the Rydberg state, this yields $R \approx 8 \mu \mathrm{m}$.

In a second step, we repeated the previous experiment but with a distance between the traps of $3.6 \pm 0.5 \mu \mathrm{m}$, which is in a regime where blockade is expected. Once again we measured the probability to excite one atom when the other one is absent and got the one-atom Rabi oscillations. When two atoms were trapped, we measured the probability to excite the two atoms simultaneously, as shown in Fig. 3b by the triangles. The simultaneous excitation of the two atoms is greatly suppressed with respect to the case where the atoms are far apart. This suppression is the signature of the blockade regime. The fact that the probability of simultaneous excitation of the two atoms is not completely cancelled may be explained by the existence of extra potential curves coming from imperfect control of the atomic state and leading to smaller interaction energies ${ }^{20}$. This imperfect control can be due to stray electric fields, imperfect polarizations of the lasers and random positions of the atoms in their trap (see last paragraph) meaning that the inter-nuclear axis is not always perfectly aligned with the quantization axis.

We now come to the direct observation of collective one-atom excitation in the blockade regime, that is, with two atoms separated by $R=3.6 \mu \mathrm{m}$. Figure 4 shows the probability to excite only one of the two atoms as a function of the duration of the excitation pulse, together with the probability to excite only one atom when the other dipole trap is empty. The two probabilities oscillate with different frequencies, the ratio of which is $1.38 \pm 0.03$ (the error corresponds to one standard deviation). This value is compatible with the ratio $\sqrt{2}$ that we expect in the blockade regime. As explained at the beginning of this letter, the oscillation of the probability to excite only one atom at a frequency $\sqrt{2} \Omega$ is the signature that the two-atom system oscillates between the state $|g, g\rangle$ and the entangled state $\left|\Psi_{+}\right\rangle=(1 / \sqrt{2})\left(\mathrm{e}^{i \mathbf{k} \cdot \mathbf{r}_{a}}|r, g\rangle+\mathrm{e}^{i \mathbf{k} \cdot \mathbf{r}_{b}}|g, r\rangle\right)$, where $\mathbf{k}=\mathbf{k}_{\mathrm{R}}+\mathbf{k}_{\mathrm{B}}$ is the sum of the wave vectors of the two lasers involved in the two-photon transition.

Finally, we analyse the influence of the atoms' motion on this entangled state. We measured a temperature of the atoms in their

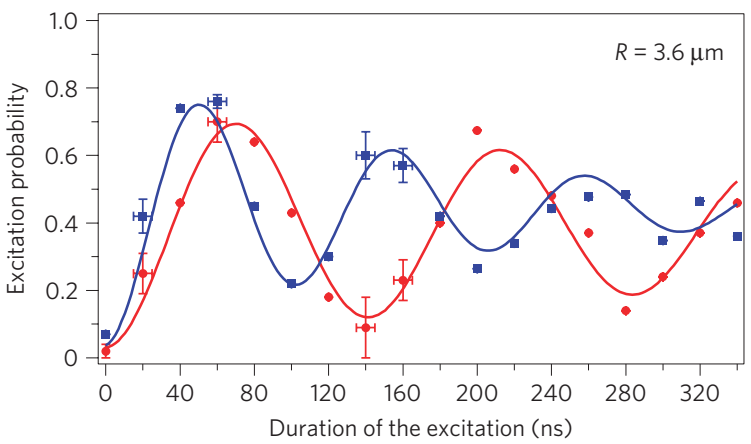

Figure 4 | Excitation of one atom versus collective excitation of two atoms separated by $\mathbf{3 . 6} \boldsymbol{\mu \mathrm { m }}$. The circles represent the probability to excite atom $a$ when atom $b$ is absent (same curve as in Fig. 3b). A fit to the data yields a frequency of this Rabi oscillation $\Omega / 2 \pi=7.0 \pm 0.2 \mathrm{MHz}$. The error comes from the fit and corresponds to one standard deviation. The squares represent the probability to excite only one atom when the two atoms are trapped and are exposed to the same excitation pulse. The fit gives an oscillation frequency $\Omega^{\prime} / 2 \pi=9.7 \pm 0.2 \mathrm{MHz}$. The ratio of the oscillation frequencies is $1.38 \pm 0.03$, close to the value $\sqrt{2}$ expected for the collective oscillation of two atoms between $|g, g\rangle$ and $\left|\Psi_{+}\right\rangle$.

trap of $70 \mu \mathrm{K}$ (ref. 23). This leads to amplitudes of the motion of $\pm 800 \mathrm{~nm}$ in the longitudinal $(y)$ direction (trap frequency $16 \mathrm{kHz}$ ) and $\pm 200 \mathrm{~nm}$ in the radial ( $x$ and $z$ ) direction of the traps (frequency $77 \mathrm{kHz}$ ). As the fastest oscillation period is $13 \mu \mathrm{s}$ and the excitation time is of the order of a hundred nanoseconds, the motion of the atoms is frozen during the excitation. The temperature results only in a dispersion of the positions of the atoms from shot to shot. Therefore, the relative phase $\phi=\mathbf{k} \cdot\left(\mathbf{r}_{a}-\mathbf{r}_{b}\right)$ between the two components of the superposition is constant during the excitation, but varies randomly from shot to shot over more than $2 \pi$. This creates an effective decoherence mechanism for the state $\left|\Psi_{+}\right\rangle$, which would prevent the direct observation of the entanglement. However, this fluctuating phase can be erased in the following way: one first couples one hyperfine ground state $|0\rangle$ to a Rydberg state $|r\rangle$, producing the state $\left|\Psi_{+}\right\rangle=(1 / \sqrt{2})\left(|r, 0\rangle+\mathrm{e}^{i \phi}|0, r\rangle\right)$ as described in this letter. Then a second pulse, applied before the atoms move, couples $|r\rangle$ to a second hyperfine ground state $|1\rangle$. If the wave vectors of the two excitations are the same, the phase during the second step cancels the phase of the first excitation. The resulting entangled state is therefore $(1 / \sqrt{2})(|1,0\rangle+|0,1\rangle)$, which involves long-lived atomic qubits ${ }^{24,25}$.

In conclusion, the results presented here indicate that we control the physical mechanism needed to deterministically entangle two atoms on fast timescales, compatible with sub-microsecond operation of a quantum gate ${ }^{1}$. Combined with our abilities to manipulate the state of a single atom ${ }^{24}$, to keep and to transport its quantum state ${ }^{25}$, our system is well adapted to applications of the Rydberg blockade in quantum-information processing.

Received 23 September 2008; accepted 11 December 2008; published online 11 January 2009

\section{References}

1. Jaksch, D. et al. Fast quantum gates for neutral atoms. Phys. Rev. Lett. 85, 2208-2211 (2000)

2. Lukin, M. D. et al. Dipole blockade and quantum information processing in mesoscopic atomic ensembles. Phys. Rev. Lett. 87, 037901 (2001).

3. Safronova, M. S., Williams, C. J. \& Clark, C. W. Optimizing the fast Rydberg quantum gate. Phys. Rev. A 67, 040303 (2003).

4. Saffman, M. \& Walker, T. G. Analysis of a quantum logic device based on dipole-dipole interactions of optically trapped Rydberg atoms. Phys. Rev. A 72, 022347 (2005).

5. Hyafil, P. et al. Coherence-preserving trap architecture for long-term control of giant Ryberg atoms. Phys. Rev. Lett. 93, 103001 (2004). 
6. Fulton, T. A. \& Dolan, G. J. Observation of single-electron charging effects in small tunnel junctions. Phys. Rev. Lett. 59, 109-112 (1987).

7. Ono, K., Austing, D. G., Tokura, Y. \& Tarucha, S. Current rectification by Pauli exclusion in a weakly coupled double quantum dot system. Science 297, 1313-1317 (2002).

8. Birnbaum, K. M. et al. Photon blockade in an optical cavity with one trapped atom. Nature 436, 87-90 (2005).

9. Cheinet, P. et al. Counting atoms using interaction blockade in an optical superlattice. Phys. Rev. Lett. 101, 090404 (2008).

10. Tong, D. et al. Local blockade of Rydberg excitation in an ultracold gas. Phys. Rev. Lett. 93, 063001 (2004).

11. Singer, K. et al. Suppression of excitation and spectral broadening induced by interactions in a cold gas of Rydberg atoms. Phys. Rev. Lett. 93, 163001 (2004).

12. Afrousheh, K. et al. Spectroscopic observation of resonant electric dipole-dipole interaction between cold Rydberg atoms. Phys. Rev. Lett. 93, 233001 (2004).

13. Cubel Liebisch, T., Reinhard, A., Berman, P. R. \& Raithel, G. Atom counting statistics ensembles of interacting Rydberg atoms. Phys. Rev. Lett. 95, 253002 (2005); erratum 98, 109903 (2007).

14. Vogt, T. et al. Dipole blockade at Förster resonances in high resolution laser excitation of Rydberg states of cesium atoms. Phys. Rev. Lett. 97, 083003 (2006).

15. van Ditzhuijzen, C. S. E. et al. Spatially resolved observation of dipole-dipole interaction between Rydberg atoms. Phys. Rev. Lett. 100, 243201 (2008).

16. Heidemann, R. et al. Rydberg excitation of Bose-Einstein condensates. Phys. Rev. Lett. 100, 033601 (2008).

17. Heidemann, R. et al. Evidence for collective Rydberg excitation in the strong blockade regime. Phys. Rev. Lett. 99, 163601 (2007).

18. Urban, E. et al. Observation of Rydberg blockade between two individual atoms. Nature Phys. 5, doi:10.1038/nphys1178 (2009).
19. Dicke, R. H. Coherence in spontaneous radiation process. Phys. Rev. 93, 99-110 (1954).

20. Walker, T. G. \& Saffman, M. Consequences of Zeeman degeneracy for the van der Waals blockade between Rydberg atoms. Phys. Rev. A 77, 032723 (2008).

21. Schlosser, N., Reymond, G., Protsenko, I. \& Grangier, P. Sub-poissonian loading of single atoms in a microscopic dipole trap. Nature 411, 1024-1027 (2001).

22. Gallagher, T. F. Rydberg Atoms (Cambridge Univ. Press, 1994).

23. Tuchendler, C., Lance, A. M., Browaeys, A., Sortais, Y. R. P. \& Grangier, P. Energy distribution and cooling of a single atom in an optical tweezer. Phys. Rev. A 78, 033425 (2008).

24. Jones, M.P.A. et al. Fast quantum state control of a single trapped neutral atom. Phys. Rev. A 75, 040301(R) (2007).

25. Beugnon, J. et al. Two-dimensional transport and transfer of a single atomic qubit in optical tweezers. Nature Phys. 3, 696-699 (2007).

\section{Acknowledgements}

We thank M. Saffman, T. Walker, R. Côté and T. F. Gallagher for enlightening discussions. We thank I. Liu for theoretical support and T. Puppe for technical assistance with the lasers. We thank C. Evellin for calculations and experimental assistance, as well as L. Servant. We acknowledge support from the EU through the IP SCALA, and from IARPA and IFRAF. A.G. is supported by a DGA fellowship. Y.M. and T.W. are supported by IFRAF.

\section{Additional information}

Supplementary Information accompanies this paper on www.nature.com/naturephysics. Reprints and permissions information is available online at http://npg.nature.com/ reprintsandpermissions. Correspondence and requests for materials should be addressed to A.B. 